\title{
The relationship of high sensitivity C-reactive protein to percent body fat mass, body mass index, waist-to-hip ratio, and waist circumference in a Taiwanese population
}

Cheng-Chieh Lin ${ }^{1,2,3,4,5+}$, Sharon LR Kardia ${ }^{6 \dagger}$, Chia-Ing Li ${ }^{2}$, Chiu-Shong Liu ${ }^{1,2,3}$, Ming-May Lai ${ }^{1,3}$, Wen-Yuan Lin ${ }^{1,3}$, Pei-Chia Chang ${ }^{7}$, Yih-Dar Lee ${ }^{8,9}$, Ching-Chu Chen ${ }^{10}$, Chih-Hsueh Lin ${ }^{1,3}$, Chuan-Wei Yang ${ }^{2}$, Chih-Yi Hsiao ${ }^{4}$, Walter Chen ${ }^{11}$, Tsai-Chung Li $i^{5,12,13^{*}}$

\begin{abstract}
Background: High-sensitivity C-reactive protein (hs-CRP) is an easily measured inflammatory biomarker. This study compared the association of percent body fat mass (\%FM), body mass index (BMI), waist circumference (WC), and waist-to-hip ratio (WHR) with hs-CRP in a Taiwanese population.

Methods: A total of 1669 subjects aged 40-88 years were recruited in 2004 in a metropolitan city in Taiwan. The relationships between obesity indicators and a high level of hs-CRP were examined using multivariate logistic regression analysis. The upper quartile of the hs-CRP distributions was defined as the high category group. The areas under the curve (AUCS) of the receiver operating characteristic curves were calculated for all obesity indicators to compare their relative ability to correctly classify subjects with a high level of hs-CRP.

Results: After multivariate adjustment, the odds ratio for \%FM was the only significant indicator that was associated with a high level of hs-CRP in men $(1.55,95 \% \mathrm{Cl}: 1.07-2.25)$. All indicators were associated with a high level of hs-CRP in women. In men, the AUCs for \%FM were significantly higher than those for BMI, WHR, and WC, when demographic and lifestyle behaviors were considered ( $p<0.001$ for all comparisons), but they were not significantly different in females.

Conclusions: Our study demonstrates that \%FM is the only obesity indicator that is strongly associated with a high level of hs-CRP after adjusting for sociodemographic factors, lifestyle behaviors and components of metabolic syndrome in both genders in a Taiwanese population aged forty years and over. In men, \%FM had the greatest ability to classify subjects with a high level of hs-CRP when only demographic and lifestyle behaviors were considered. Our study finding has important implications for the screening of obesity in community settings.
\end{abstract}

\section{Background}

Obesity is a major public health problem in the world, affecting people in both developed and developing countries [1-4]. According to reports, there are about 250 million adults suffering from obesity [5,6]. A previous survey has shown that the age-adjusted prevalence of

\footnotetext{
* Correspondence: tcli@mail.cmu.edu.tw

† Contributed equally

${ }^{5}$ Department of Healthcare Administration, College of Health Science, Asia University, Taichung, Taiwan

Full list of author information is available at the end of the article
}

obesity is rapidly increasing in Taiwan, from $10.5 \%$ to $15.9 \%$ for men from 1993-1996 to 2000-2001 [7]. Of most importance, obesity is associated with numerous chronic health conditions or diseases, such as diabetes, hypertension, and cardiovascular disease $[8,9]$.

High-sensitivity C-reactive protein (hs-CRP), the main acute phase protein in humans, is a sensitive marker for systemic inflammation. Previous cross-sectional studies have shown that elevated hs-CRP levels correlate significantly with features of metabolic abnormality, including adiposity, hyperinsulinemia, insulin resistance,

\section{Biomed Central}


hypertriglyceridemia, and low high-density lipoprotein cholesterol (HDL-C) [10-13]. Although the physiological mechanisms linking elevated hs-CRP to these disorders are not known, it is possible that the association is partly mediated by adipose tissue, a main source of inflammatory cytokines. Most epidemiologic studies identifying strong associations between hs-CRP and obesity indicators predominantly use anthropometric indexes [10-13]. Only a few studies have used measures of body fat [14-16].

Body mass index (BMI) is an indicator of heaviness rather than fatness, and cannot distinguish body fat from fat-free mass. Waist-to-hip ratio (WHR) is a measure of regional fat distribution, whereas waist circumference (WC) is a measure of central obesity. Bio-electrical impedance analysis (BIA) produces a close estimate of fat-free mass throughout a wide range of body composition [17]. BIA is a non-invasive measurement of body composition and is especially useful in large epidemiologic studies. It also possesses many advantages over other methods, in that it is inexpensive, simple, fast, safe, portable, easy-to-perform, and requires minimal operator training $[18,19]$. The current study was designed to explore the values of obesity indicators of percent body fat mass (\%FM), as determined by BIA for the total body, compared to BMI, WC, and WHR, in terms of their independent relation to hs-CRP under two conditions: one considering metabolic syndrome (MetS)'s components, except obesity, and the other not considering these MetS components.

\section{Methods \\ Participants}

This was a community-based cross-sectional study based on data from the Taichung Community Health Study. The detailed methodology has been described elsewhere [20-25]. A total of 2,359 residents of Taichung City, Taiwan, aged 40 and over, participated in the study in October 2004. Hs-CRP levels were measured only for the first 1669 consecutive subjects. This study was approved by the Human Research Committee of China Medical University Hospital. Written informed consent was obtained from each participant.

\section{Anthropometric measurements and laboratory examinations}

Anthropometric measurements and blood samples were obtained from the complete physical examination. Weight and height were measured on an auto-anthropometer (super-view, HW-666), with the subjects shoeless and wearing light clothing. BMI was derived from the formula, weight $(\mathrm{kg}) \div(\text { height })^{2}\left(\mathrm{~m}^{2}\right)$. With the participant standing, WC was measured midway between the superior iliac crest and the costal margin, and hip circumference at its maximum protrusion point of the buttocks around the pelvis, and then the WHR was calculated as a measure of regional fat distribution. \%FM was assessed by a body composition analyzer (Tanita BC-418, Arlington Heights, Illinois, USA). The measurements were performed with the subjects stepping onto the measuring platform without shoes and after wiping the soles of their feet. The amount of body fat was expressed as a percentage of total weight. Previous studies have confirmed the validity of BIA in estimating body composition compared to dual energy X-ray absorptiometry (DEXA) $[19,26]$. The means values for BIA and DEXA were very close [26]. BIA was a good predictor of DEXA-derived fat-free mass $(\mathrm{r}=0.85-0.88)$ and was superior to BMI in measuring body fat [19]. The principle of BIA is that fat tissue exhibits greater resistance to the flow of electrical current than fat-free tissue because of differences in water content.

Blood pressure was measured by an electronic device (COLIN, VP-1000, Japan) three times after the subjects had rested for 20 minutes. The lowest systolic and diastolic blood pressure was recorded. Blood was drawn from an antecubital vein in the morning after a 12-hour overnight fasting and was sent for analysis within four hours of blood collection. Hs-CRP levels were measured by nephelometry, a latex particle-enhanced immunoassay (TBA-200FR, Tokyo, Japan). The interassay and intra-assay CVs were $<2.0 \%$ and $<1.9 \%$, respectively. The lower detection limit of the assay was $0.1 \mathrm{mg} / \mathrm{L}$. Biochemical markers such as fasting plasma glucose, HDL-C, and triglyceride were analyzed by a biochemical autoanalyzer (Beckman Coulter Synchron system, Lx-20, Fullerton, CA, USA) at the Clinical Laboratory Department of China Medical University Hospital. Fasting plasma glucose was measured in blood obtained by use of NAF TUBE. NAF TUBE contains $5 \mathrm{mg}$ sodium fluoride to inhibit glucose metabolism and $4 \mathrm{mg}$ potassium oxalate to chelate calcium and prevent coagulation. The interassay and intra-assay CVs for fasting plasma glucose were $4 \%$ and $4 \%$, respectively. We measured cholesterol and triglyceride in serum mode. A SST tube was used. The SST ${ }^{\mathrm{Tx}}$ tube refers to the Serum Separator Tube containing clot activator and serum separator gel. The silica particles that coat the walls of the BD Vacutainer ${ }^{\circ} \mathrm{SST}^{\mathrm{m}}$ tube are the clot activator. Initial activation occurs when blood enters the tube and contacts the particles on the tube wall. Triglyceride levels were determined using an enzymatic colorimetric method. The interassay and intra-assay CVs for triglyceride were 6.8\% and $5 \%$, respectively. The HDL-C level was measured by a direct HDL-C method and the interassay and intraassay CVs were $4.5 \%$ and $4.5 \%$, respectively. The lowdensity lipoprotein cholesterol (LDL-C) level was also measured using a direct LDL-C method, and interassay and intra-assay CVs were $4.5 \%$ and $3 \%$, respectively. The 
serum insulin level was measured by a commercial enzyme-linked immunosorbent assay kit (Diagnostic Products, Los Angeles, CA). The interassay CV for insulin was $8.7 \%$ and the intra-assay $\mathrm{CV}$ was $3.4 \%$. Insulin sensitivity was estimated with a Homeostasis Model Assessment (HOMA-IR) equation. The HOMA-IR equals fasting serum insulin $(\mu \mathrm{U} / \mathrm{ml})$ times fasting plasma glucose (mmol/l) divided by 22.5 [27]. The cut-offs defining abnormality of triglycerides, HDL-C, blood pressure, and fasting glucose were in accordance with the AHA/NHLBI statement that was used to define the four components of MetS [28]: elevated triglycerides ( $\geq 150 \mathrm{mg} / \mathrm{dL}$ ), reduced HDL-C ( $<40 \mathrm{mg} / \mathrm{dL}$ for men, $<50 \mathrm{mg} / \mathrm{dL}$ for women), elevated blood pressure (BP $\geq 130 / \geq 85 \mathrm{mmHg}$ ), and elevated fasting glucose $(\geq 100 \mathrm{mg} / \mathrm{dL})$.

Data regarding smoking, alcohol drinking, physical activity, betel nut chewing and family history of cardiovascular-related diseases were collected by questionnaire when the participants underwent a complete physical examination. Subjects who self-reported any of these characteristics (smoking, alcohol drinking, betel nut chewing and family history of cardiovascular-related diseases) were placed into groups based on the specific characteristic. Those in the non-smoking group had never smoked or had smoked less than 100 cigarettes during their lifetime, whereas those in smoking group smoked currently or had smoked more than or equal to 100 cigarettes during their lifetime. Individuals who selfreported alcohol drinking, betel nut chewing or exercise were classified into the group with this specific characteristic. Those whose parents or siblings had a specific cardiovascular-related disease were classified as having a family history of the disease.

\section{Statistical analysis}

Continuous variables were reported as means, whereas categorical variables were reported as numbers (percentage). The cutoff points for WC [28] were $90(\mathrm{~cm})$ for men and $80(\mathrm{~cm})$ for women, and for WHR [29] were 0.9 in men and 0.85 in women. The value of the cutoff point of BMI for defining overweight was 24 [30]. We defined the subjects in the upper quartile of the \%FM and hs-CRP distributions as the high category group. To examine the relationship between obesity indicators and hs-CRP, crude odds ratios (ORs) were first used; then, multivariate logistic regression analysis was used to investigate the independent effect on the high level of hs-CRP, adjusting for age, smoking, alcohol drinking, and betel nut chewing. Finally, variables for abnormality of the MetS components other than obesity were entered into models. ORs and their $95 \%$ confidence intervals were calculated. The areas under curve (AUCs) for each receiver operating characteristics curve were calculated to compare the relative ability of obesity indicators to correctly classify subjects with a high level of hs-CRP, and the nonparametric method was used to test whether the AUCs of these four obesity indicators were different [31]. All analyses were conducted using SAS version 9.1 (SAS Institute Inc, Cary, NC). A two-sided significant level of $\mathrm{p}<0.05$ was reported.

\section{Results}

A total of 1669 subjects (807 men and 862 women) were analyzed in the final model. The sociodemographic and anthropometric characteristics and biomarkers of the obese participants, as defined by \%FM, BMI, WHR, and WC, and stratified by gender, are summarized in Tables 1 and 2. Obese participants, both men and women, identified by these four obesity indicators had similar characteristics.

Table 3 shows the ORs of the high level of hs-CRP for each obesity indicator for men and women. Without considering any covariates, all ORs were significant, except BMI, and ORs were largest for \%FM for both men and women (OR: 1.94, 95\% CI: 1.37-2.73 in men; OR: 3.59 , 95\% CI: 2.59-4.98 in women). After adjusting for age, smoking status, alcohol drinking, and betel nut chewing, the effect of \%FM still remained significant (OR: $1.89,95 \%$ CI: $1.33-2.68$ in men; OR: $3.34,95 \% \mathrm{CI}$ : 2.39-4.68 in women). After further adjusting for hyperglycemia, hypertriglyceridemia, hypertension, and low HDL-C, the OR for \%FM became attenuated, but remained significant $(1.55,95 \% \mathrm{CI}: 1.07-2.25$ in men; OR: $2.89,95 \%$ CI: $2.03-4.11$ in women). In women, the crude ORs for the four obesity indicators were all statistically significant. These ORs remained significant after considering age, smoking, alcohol drinking, betel nut chewing, and the four components of MetS. The high categories of BMI, WHR, and WC were associated with a 2.11 -fold (95\% CI: 1.51-2.95), 2.07-fold (1.45-2.97) and 2.37-fold (1.67-3.35) increased risk of having a high level of hs-CRP, respectively.

The AUCs and 95\% CIs of four obesity indicators stratified by gender are shown in Table 4 . In men, the AUCs for BMI, WC, and WHR were similar and were all significantly lower than that for \%FM $\left(\chi^{2}\right.$ for overall test $=14.77, \mathrm{p}=0.002$, the $\mathrm{p}$ values for pair-wise comparisons were all $<0.001$, Figure 1$)$. On the other hand, in women, the AUCs for \%FM, BMI, and WC were similar and were slightly higher than that for WHR, but there were no significant differences between the AUCs for the different obesity indicators $\left(\chi^{2}=5.55, \mathrm{p}=\right.$ 0.1355 , Figure 2). After further adjusting for the four components of MetS, the differences between the AUCs for the four obesity indicators were no longer statistically significant in men. 
Table 1 Prevalence of cardiovascular risk factors in obese individuals as defined by each obesity indicator, and stratified by gender, $\mathrm{n}(\%)$

\begin{tabular}{|c|c|c|c|c|c|c|c|c|}
\hline \multirow[b]{3}{*}{ Variables } & \multicolumn{4}{|c|}{ Male } & \multicolumn{4}{|c|}{ Female } \\
\hline & \multicolumn{4}{|c|}{ Obesity defined by } & \multicolumn{4}{|c|}{ Obesity defined by } \\
\hline & $\begin{array}{c}\text { \%FM } \\
(\geqq 29.6)\end{array}$ & $\begin{array}{l}\text { BMI } \\
(\geqq 24)\end{array}$ & $\begin{array}{l}\text { WHR } \\
(\geqq 0.9)\end{array}$ & $\begin{array}{c}W C \\
(\geqq 90 \mathrm{~cm})\end{array}$ & $\begin{array}{c}\text { \%FM } \\
(\geqq 40.1)\end{array}$ & $\begin{array}{l}\text { BMI } \\
(\geqq 24)\end{array}$ & $\begin{array}{c}\text { WHR } \\
(\geqq 0.85)\end{array}$ & $\begin{array}{c}W C \\
(\geqq 80 \mathrm{~cm})\end{array}$ \\
\hline Obesity & $203(25.15)$ & $478(59.23)$ & $340(42.13)$ & $240(29.74)$ & $222(25.75)$ & $366(42.46)$ & $189(21.93)$ & $250(29.00)$ \\
\hline Smoking $^{a}$ & $65(32.02)$ & 139 (29.08) & $118(34.81)$ & $80(33.33)$ & $8(3.62)$ & $10(2.74)$ & $6(3.17)$ & $7(2.80)$ \\
\hline Drinking $^{b}$ & 77 (37.93) & 189 (39.54) & $128(37.76)$ & $98(40.83)$ & $16(7.24)$ & $27(7.40)$ & $15(7.94)$ & $17(6.80)$ \\
\hline Betel nut chewing ${ }^{b}$ & $14(6.90)$ & $37(7.76)$ & $27(8.01)$ & $17(7.08)$ & $0(0.00)$ & $0(0.00)$ & $0(0.00)$ & $0(0.00)$ \\
\hline Exercise $^{\mathrm{b}}$ & $135(66.50)$ & $320(66.95)$ & $221(65.19)$ & $164(68.33)$ & $135(60.81)$ & $236(64.48)$ & $121(64.02)$ & $155(62.00)$ \\
\hline Family history of diabetes ${ }^{c}$ & $48(23.65)$ & $116(24.27)$ & $72(21.18)$ & $53(22.08)$ & $52(23.42)$ & $98(26.78)$ & $46(24.34)$ & $58(23.20)$ \\
\hline Hyperglycemia & $111(54.68)$ & $231(48.33)$ & $168(49.41)$ & $131(54.58)$ & $97(43.69)$ & $153(41.80)$ & $86(45.50)$ & $114(45.60)$ \\
\hline Hypertriglyceridemia & $102(50.25)$ & $183(38.28)$ & $143(42.06)$ & $104(43.33)$ & $64(28.83)$ & $101(27.60)$ & $62(32.80)$ & $74(29.60)$ \\
\hline Hypertension & $174(85.71)$ & $354(74.06)$ & $264(77.65)$ & $201(83.75)$ & $163(73.42)$ & $244(66.67)$ & $129(68.25)$ & $185(74.00)$ \\
\hline Low HDL cholesterol & $136(67.00)$ & $301(62.97)$ & $212(62.35)$ & $151(62.92)$ & 157 (70.72) & $248(67.76)$ & $131(69.31)$ & $171(68.40)$ \\
\hline High level of hs-CRP & $74(36.45)$ & $137(28.66)$ & $104(30.59)$ & $76(31.67)$ & $105(47.30)$ & $140(38.25)$ & $83(43.92)$ & $109(43.60)$ \\
\hline
\end{tabular}

\%FM: percent body fat mass, BMI: body mass index, WHR: waist-to-hip ratio, WC: waist circumference, high-density lipoprotein (HDL), high-sensitivity C-reactive protein (hs-CRP).

a: Those in the non-smoking group had never smoked or had smoked less than 100 cigarettes during their lifetime, whereas those in the smoking group smoked currently or had smoked more than or equal to 100 cigarettes during their lifetime.

b: Individuals who self-reported the characteristics of alcohol drinking, betel chewing or exercise were classified into the group with this specific characteristic.

c: Those whose parents or siblings had diabetes were classified as having a family history of diabetes.

Table 2 Distributions of sociodemographic and biochemical characteristics in obese individuals defined by each obesity indicator, and stratified by gender

\begin{tabular}{|c|c|c|c|c|c|c|c|c|}
\hline \multirow[b]{3}{*}{ Variables } & \multicolumn{8}{|c|}{ Obesity defined by* } \\
\hline & \multicolumn{2}{|c|}{$\% F M$} & \multicolumn{2}{|c|}{ BMI } & \multicolumn{2}{|c|}{ WHR } & \multicolumn{2}{|c|}{ WC } \\
\hline & Mean & SD & Mean & SD & Mean & SD & Mean & SD \\
\hline \multicolumn{9}{|l|}{ Male } \\
\hline Age (years) & 59.6 & 12.7 & 57.4 & 12.0 & 60.1 & 12.4 & 59.5 & 12.4 \\
\hline Fasting blood glucose (mmol/L) & 6.1 & 1.7 & 5.9 & 1.6 & 6.1 & 1.9 & 6.1 & 1.8 \\
\hline Fasting insulin (pmol/L) & 96.6 & 61.0 & 75.4 & 52.1 & 76.1 & 51.2 & 91.9 & 58.1 \\
\hline HOMA-IR & 3.7 & 2.7 & 2.8 & 2.3 & 2.9 & 2.4 & 3.6 & 2.7 \\
\hline Triglyceride (mmol/L) & 1.8 & 1.0 & 1.7 & 1.4 & 1.8 & 1.40 & 1.8 & 1.5 \\
\hline HDL-cholesterol (mmol/L) & 1.0 & 0.2 & 1.0 & 0.2 & 1.0 & 0.2 & 1.0 & 0.3 \\
\hline LDL-cholesterol (mmol/L) & 3.5 & 0.9 & 3.3 & 0.8 & 3.4 & 0.9 & 3.4 & 0.9 \\
\hline Diastolic blood pressure $(\mathrm{mmHg})$ & 86.1 & 8.8 & 83.5 & 10.1 & 83.9 & 9.8 & 84.9 & 9.1 \\
\hline Systolic blood pressure (mmHg) & 144.9 & 17.8 & 139.2 & 19.1 & 141.2 & 19.2 & 142.9 & 18.1 \\
\hline $\mathrm{Hs}-\mathrm{CRP}(\mathrm{mg} / \mathrm{l})^{\#}$ & 0.18 & 1.05 & 0.16 & 1.03 & 0.17 & 1.04 & 0.17 & 1.05 \\
\hline \multicolumn{9}{|l|}{ Female } \\
\hline Age (years) & 58.8 & 10.2 & 57.0 & 9.7 & 58.4 & 11.5 & 58.7 & 10.6 \\
\hline Fasting blood glucose (mmol/L) & 6.1 & 1.9 & 5.9 & 1.8 & 6.0 & 1.8 & 6.1 & 2.0 \\
\hline Fasting insulin (pmol/L) & 82.4 & 54.4 & 74.5 & 55.6 & 83.6 & 65.7 & 79.2 & 60.4 \\
\hline HOMA-IR & 3.2 & 2.6 & 2.9 & 2.8 & 3.3 & 3.4 & 3.2 & 3.2 \\
\hline Triglyceride (mmol/L) & 1.5 & 0.9 & 1.4 & 0.8 & 1.5 & 1.0 & 1.5 & 0.9 \\
\hline HDL-cholesterol (mmol/L) & 1.2 & 0.3 & 1.2 & 0.3 & 1.2 & 0.3 & 1.2 & 0.3 \\
\hline LDL-cholesterol (mmol/L) & 3.5 & 0.8 & 3.5 & 0.8 & 3.4 & 0.8 & 3.4 & 0.8 \\
\hline Diastolic blood pressure $(\mathrm{mmHg})$ & 80.8 & 11.6 & 79.0 & 11.7 & 79.1 & 11.3 & 80.1 & 11.3 \\
\hline Systolic blood pressure (mmHg) & 143.3 & 21.9 & 139.3 & 21.6 & 139.7 & 22.6 & 142.0 & 22.0 \\
\hline $\mathrm{Hs}-\mathrm{CRP}(\mathrm{mg} / \mathrm{ll})^{\#}$ & 0.21 & 1.05 & 0.18 & 1.04 & 0.20 & 1.05 & 0.21 & 1.05 \\
\hline
\end{tabular}

\%FM: percent body fat mass, BMI: body mass index, WHR: waist-to-hip ratio, WC: waist circumference, homeostasis model assessment of insulin resistance (HOMA-IR), high-density lipoprotein (HDL), low-density lipoprotein (LDL), high-sensitivity C-reactive protein (hs-CRP).

\#: geometric mean was presented.

*The cutoff values for $\% F M$ : $\geq 29.6$ in men and $\geq 40.1$ in women; for BMI: $\geq 24$; for WHR: $\geq 0.9$ in men and $\geq 0.85$ in women; for WC: $\geq 90 \mathrm{~cm}$ in men and $\geq 80$ in women. 
Table 3 The odds ratios of a high level of hs-CRP for obesity as defined by \%FM, BMI, WHR, and WC ${ }^{\mathrm{a}}$

\begin{tabular}{|c|c|c|}
\hline \multirow[b]{2}{*}{ Univariate model } & \multicolumn{2}{|c|}{ OR (95\%) } \\
\hline & Men & Women \\
\hline BMl & $1.36(0.98,1.88)$ & $2.68(1.97,3.66)$ \\
\hline WHR & $1.47(1.07,2.01)$ & $2.73(1.94,3.84)$ \\
\hline WC & $1.47(1.05,2.05)$ & $3.04(2.21,4.18)$ \\
\hline$\% \mathrm{FM}$ & $1.94(1.37,2.73)$ & $3.59(2.59,4.98)$ \\
\hline \multicolumn{3}{|l|}{ Multivariate model§ } \\
\hline $\mathrm{BMl}$ & $1.45(1.04,2.02)$ & $2.56(1.86,3.50)$ \\
\hline WHR & $1.26(0.90,1.74)$ & $2.50(1.77,3.54)$ \\
\hline WC & $1.42(1.01,1.99)$ & $2.81(2.02,3.90)$ \\
\hline$\% F M$ & $1.89(1.33,2.68)$ & $3.34(2.39,4.68)$ \\
\hline \multicolumn{3}{|l|}{ Multivariate model\& } \\
\hline BMl & $1.25(0.87,1.77)$ & $2.11(1.51,2.95)$ \\
\hline WHR & $1.06(0.76,1.50)$ & $2.07(1.45,2.97)$ \\
\hline WC & $1.19(0.83,1.70)$ & $2.37(1.67,3.35)$ \\
\hline$\% \mathrm{FM}$ & $1.55(1.07,2.25)$ & $2.89(2.03,4.11)$ \\
\hline \multicolumn{3}{|l|}{ Multivariate model\&f } \\
\hline BMl & $1.12(0.93,1.33)$ & $1.65(1.39,1.97)$ \\
\hline WHR & $1.09(0.90,1.31)$ & $1.40(1.19,1.66)$ \\
\hline WC & $1.18(0.98,1.41)$ & $1.76(1.48,2.12)$ \\
\hline$\% F M$ & $1.50(1.25,1.80)$ & $1.72(1.42,2.10)$ \\
\hline
\end{tabular}

\%FM: percent body fat mass; BMI: body mass index; WHR: waist-to-hip ratio; WC: waist circumference.

a: The cutoff values for $\% F M: ~ \geq 29.6$ in men and $\geq 40.1$ in women; for BMl: $\geq 24$; for WHR: $\geq 0.9$ in men and $\geq 0.85$ in women; for WC: $\geq 90 \mathrm{~cm}$ in men and $\geq 80$ in women.

$\S$ : Logistic regression adjusted for age, smoking, alcohol drinking and betel nut chewing

\&: Logistic regression adjusted for age, smoking, alcohol drinking, betel nut chewing, hyperglycemia, hypertriglyceridemia, hypertension and low HDL cholesterol.

f: per 1-SD increment.

\section{Discussion}

In this community-based study, we demonstrated that all obesity indicators, except WHR in men, were strongly associated with a high CRP level in Taiwanese men and women when sociodemographic factors and lifestyle behaviors were considered. Only the association for \%FM remained significant in women after further considering individual abnormalities in the four components of MetS.

Hs-CRP is an easily measured inflammatory biomarker and is released by the liver under the stimulation of cytokines, including interleukin-6, interleukin-1, and tumor necrosis factor-alpha. It has been shown hs-CRP has associations with endothelial dysfunction and insulin resistance syndrome [32]. Although a relationship has been found between hs-CRP and DEXA-measured trunk fat [33], our finding is the first to show that hs-CRP is only associated with \%FM in men and women compared with BMI, WHR, and WC, indicating that \%FM is the
Table 4 Comparisons of AUCs for percent body fat mass, body mass index, waist-to-hip ratio, and waist circumference, stratified by gender

\begin{tabular}{lcccccc}
\hline & \multicolumn{2}{c}{ Male } & \multicolumn{3}{c}{ Female } \\
& AUC & $\mathbf{9 5 \%}$ Cl & $\boldsymbol{x}^{\mathbf{2}}$ & AUC & $\mathbf{9 5 \%} \mathbf{C l}$ & $\boldsymbol{x}^{\mathbf{2}}$ \\
\hline ROC curve & & & $14.77^{* * a}$ & & & 5.55 \\
\%FM & 0.67 & $0.62,0.71$ & & 0.69 & $0.65,0.73$ & \\
BMI & 0.62 & $0.57,0.66$ & & 0.69 & $0.65,0.73$ & \\
WHR & 0.61 & $0.57,0.66$ & & 0.65 & $0.61,0.70$ & \\
WC & 0.62 & $0.58,0.67$ & & 0.69 & $0.65,0.73$ & \\
ROC curve\& & & & 7.74 & & & 6.81 \\
\%FM & 0.68 & $0.63,0.72$ & & 0.71 & $0.67,0.75$ & \\
BMI & 0.65 & $0.60,0.69$ & & 0.71 & $0.67,0.75$ & \\
WHR & 0.64 & $0.60,0.69$ & & 0.69 & $0.65,0.73$ & \\
WC & 0.65 & $0.60,0.69$ & & 0.71 & $0.67,0.75$ & \\
\hline
\end{tabular}

\%FM: percent body fat mass; BMI: body mass index; WHR: waist-to-hip ratio; WC: waist circumference, AUC: area under curve.

**: $p<0.01$;

a: AUC of ROC curve for \%FM was significant larger than those for BMI, WHR, and WC.

§: Logistic regression adjusted for age, smoking, alcohol drinking and betel nut chewing.

\&: Logistic regression adjusted for age, smoking, alcohol drinking, betel nut chewing, hyperglycemia, hypertriglyceridemia, hypertension and low HDL cholesterol.

obesity indicator that can capture the inflammatory phenomena in both genders that are responsible for the higher likelihood of diabetes and cardiovascular events. In addition, \%FM had a greater ability to classify subjects with a high level of hs-CRP compared with BMI, WHR, and WC in men, when demographic and lifestyle behaviors were considered, but this ability diminished when MetS components were considered. This finding has important implications for obesity screening in community surveys. The BIA measurement may not be more valuable than the other obesity indicators when health surveys use blood samples, but is a measurement worth using when the health survey for screening obesity does not draw blood samples.

WHR has been the traditional anthropometric index for assessing central adiposity; however, the use of WC is gaining support [34-37] and popularity as an alternative, simpler option [38-40]. WHR was the only obesity indicator that did not perform well in men in identifying individuals with a high hs-CRP level after adjusting for age, smoking, alcohol drinking, and betel nut chewing. In Craig's work, WHR did not perform well in identifying risk factors for cardiovascular disease and undiagnosed diabetes [41]. In addition, the correlations with individual risk factors were weaker than those of other measures of body composition, especially in women [41]. On the other hand, WHR explained the highest percentage of the variability of CRP in men in Thorand's work [15], and WHR was significantly correlated with CRP in both men and women [16]. The possible 


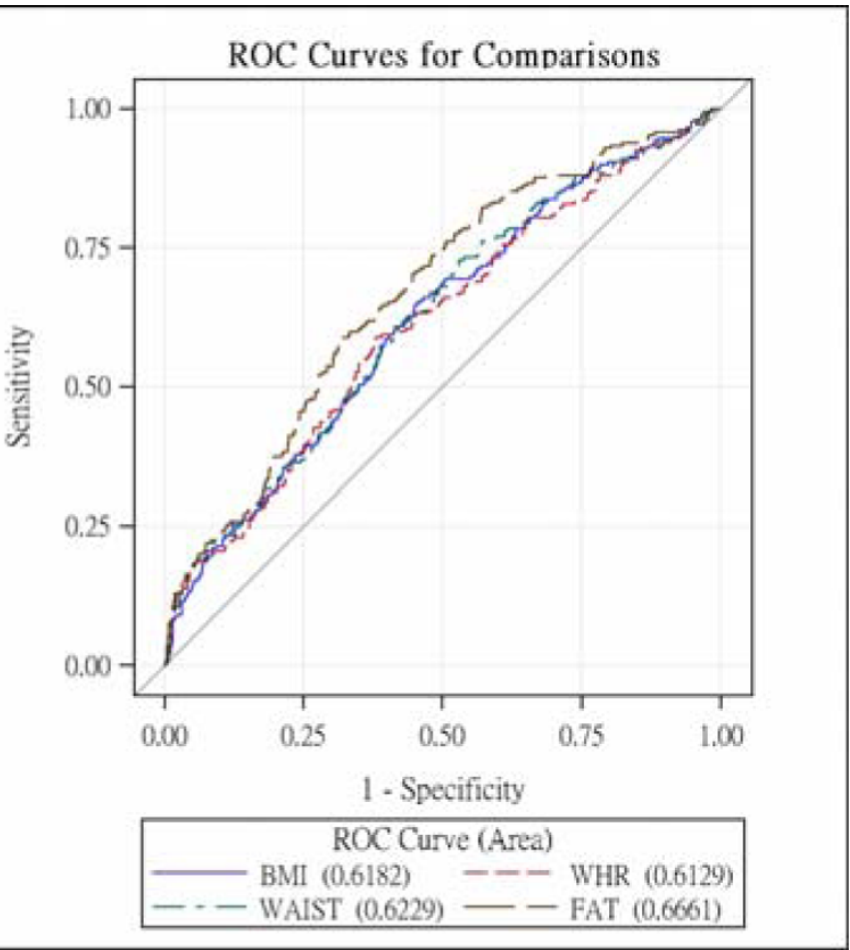

Figure 1 The receiver operating characteristics curves of BMI, WHR, WC and \%FM for males.

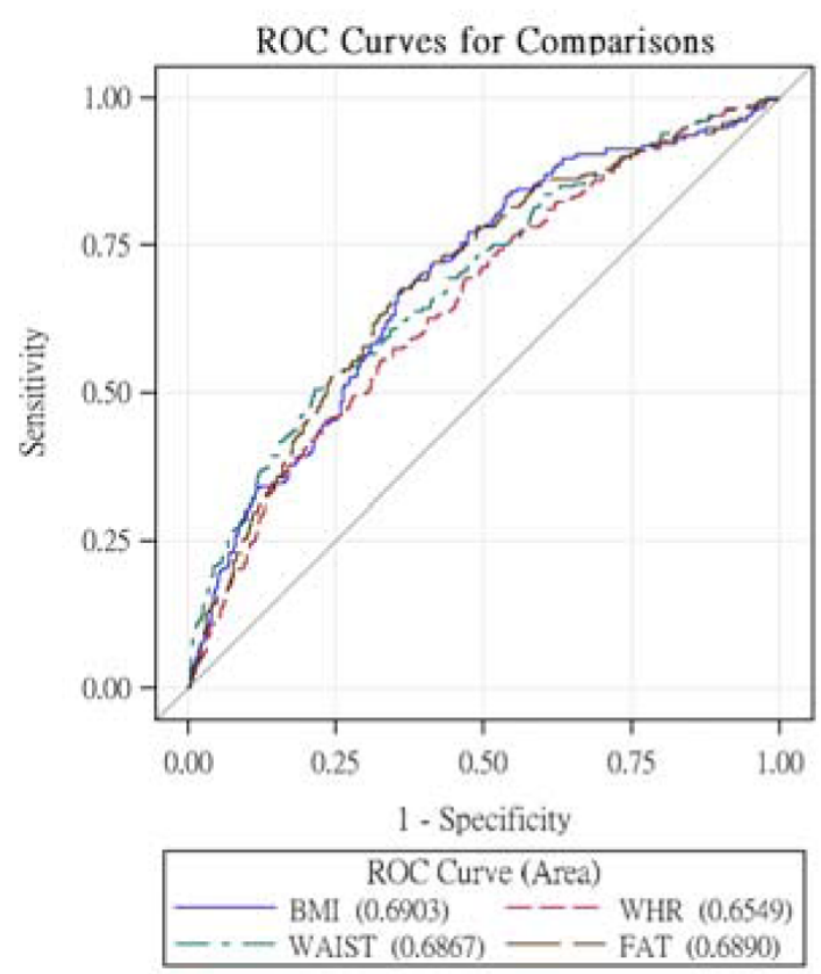

Figure 2 The receiver operating characteristics curves of BMI, WHR, WC and \%FM for females. 
explanation for the differences in the findings among these studies was that these studies were conducted with different ethnic groups, or the populations in these studies had different levels or types of obesity.

The limitations of this study must be considered. The principal limitation relevant to the interpretation of our results is the use of cross-sectional data; thus, causal pathways underlying the observed relationships cannot be inferred. Second, these analyses were restricted to the first 1669 subjects entering the current study, indicating that potential selection bias might exist. To assess this possibility, we examined the demographic characteristics of the individuals with and without hs-CRP measurement by comparing age, sex, and administrative unit, and similar distributions were found. The nondifferential distributions in age, sex, and administrative unit indicate this kind of selection error might be random, thus, the biased results in the effect may be toward the null, a lesser threat to validity.

\section{Conclusion}

In summary, this study demonstrates that \%FM is strongly associated with a high level of hs-CRP in both genders in a Taiwanese population aged forty years and over. The novelty of the study is that \%FM is the only significant indicator related to hs-CRP among various anthrometric indexes in men when demographic and lifestyle behaviors, and the individual components of MetS are considered. This finding highlights the importance of \%FM in screening obese individuals who have a higher likelihood of chronic inflammation, especially when no blood sample is drawn. BIA measurement offers a convenient and practical approach to body composition assessment and may be useful and valuable in community settings. Our study finding has important implications for the screening of obesity.

\section{Abbreviations \\ hs-CRP: high-sensitivity C-reactive protein; HDL-C: high-density lipoprotein cholesterol; BMI: body mass index; WHR: waist-to-hip ratio; WC: waist circumference; BIA: bio-electrical impedance analysis; \%FM: percent body fat mass; DEXA: dual energy X-ray absorptiometry; MetS: metabolic syndrome; ORs: odds ratios; AUCs: receiver operating characteristics curve.}

\section{Acknowledgements}

This study was supported by grants from the National Science Council of Taiwan (NSC93-2314-B-039-025\&NSC94-2314-B-039-024) and Taiwan Department of Health Clinical Trial and Research Center of Excellence (DOH99-TD-B-111-004).

\section{Author details}

'Department of Family Medicine, China Medical University \& Hospital, Taichung, Taiwan. ${ }^{2}$ Medical Research, China Medical University \& Hospital, Taichung, Taiwan. ${ }^{3}$ Department of Family Medicine, College of Medicine, China Medical University \& Hospital, Taichung, Taiwan. ${ }^{4}$ Institute of Health Care Administration, College of Public Health, China Medical University \& Hospital, Taichung, Taiwan. ${ }^{5}$ Department of Healthcare Administration, College of Health Science, Asia University, Taichung, Taiwan. ${ }^{6}$ Department of
Epidemiology, University of Michigan, Ann Arbor, Michigan, USA. ${ }^{7}$ Administration Center, China Medical University \& Hospital, Taichung, Taiwan. ${ }^{8}$ Department of Psychiatry, Medical College, National Cheng-Kung University, Tainan, Taiwan. ${ }^{9}$ Bristol-Myers Squibb (Taiwan) Ltd, Global Development \& Medical Affairs, Taipei, Taiwan. ${ }^{10}$ Division of Endocrinology and Metabolism, Department of Medicine, China Medical University \& Hospital, Taichung, Taiwan. ${ }^{11}$ Department of Medicine, China Medical University \& Hospital, Taichung, Taiwan. ${ }^{12}$ Graduate Institute of Biostatistics \& Chinese Medicine Science, China Medical University \& Hospital, Taichung, Taiwan. ${ }^{13}$ Biostatistics Center, China Medical University \& Hospital, Taichung, Taiwan.

\section{Authors' contributions}

CCL, SLRK and TCL contributed equally to the design of the study and the direction of its implementation, including supervision of the field activities, quality assurance and control. CIL, CSL, WYL, MML, CCC, TL, CYH, WC and PCC supervised the field activities. CSL and YDL helped conduct the literature review and prepare the Methods and the Discussion sections of the text. TCL, SLRK, CIL, CSL, CCL, CHL and CWY designed the study's analytic strategy and conducted the data analysis. All authors read and approved the final manuscript.

\section{Competing interests}

The authors declare that they have no competing interests.

Received: 7 January 2010 Accepted: 28 September 2010 Published: 28 September 2010

\section{References}

1. Kannel WB, D'Agostino RB, Cobb JL: Effect of weight on cardiovascular disease. Am J Clin Nutr 1996, 63(Suppl 4):19-22.

2. Carroll KK: Obesity as a risk factor for certain types of cancer. Lipids 1998, 33:1055-1059.

3. Alberti $K G$, Zimmet $P Z$ : Definition, diagnosis and classification of diabetes mellitus and its complications. Part 1: diagnosis and classification of diabetes mellitus provisional report of a WHO consultation. Diabet Med 1998, 15:539-553.

4. WHO: Obesity: Preventing and managing the global epidemic. Geneva: WHO 1998.

5. Kuczmarki RJ, Flegal KM, Campbell SM, Johnson CL: Increasing prevalence of overweight among US adults. The National Health and Nutrition Examination Surveys, 1960 to 1991. JAMA 1998, 22:39-47.

6. Seidell JC, Verschu Ren WM, Van Leer EM, Kromhout D: Overweight, underweight, and mortality: a prospective study of 48,287 men and women. Arch Intern Med 1996, 14:1132-1143.

7. Chu NF: Prevalence of obesity in Taiwan. Obesity Reviews 2005, 6:271-274.

8. Must A, Spadano J, Coakley EH, Field AE, Colditz G, Dietz WH: The disease burden associated with overweight and obesity. JAMA 1999, 282:1523-1529.

9. Kopelman PG: Obesity as a medical problem. Nature 2000, 404:635-643.

10. Frohlich M, Imhof A, Berg G, Hutchinson WL, Pepys MB, Boeing H, Muche R, Brenner $\mathrm{H}$, Koenig W: Association between C-reactive protein and features of the metabolic syndrome: a population-based study. Diabetes Care 2000, 23:1835-1839.

11. Hak AE, Stehouwer CD, Bots ML, Polderman KH, Schalkwijk CG, Westendorp IC, Hofman A, Witteman JC: Associations of C-reactive protein with measures of obesity, insulin resistance, and subclinical atherosclerosis in healthy, middle-aged women. Arterioscler Thromb Vasc Biol 1999, 19:1986-1991.

12. Chambers JC, Eda S, Bassett P, Karim Y, Thompson SG, Gallimore JR, Pepys MB, Kooner JS: C-reactive protein, insulin resistance, central obesity, and coronary heart disease risk in Indian Asians from the United Kingdom compared with European whites. Circulation 2001, 104:145-150.

13. Mendall MA, Patel P, Ballam L, Strachan D, Northfield TC: C reactive protein and its relation to cardiovascular risk factors: a population based cross sectional study. BMJ 1996, 312:1061-1065.

14. Greenfield JR, Samaras K, Jenkins AB, Kelly PJ, Spector TD, Gallimore JR, Pepys MB, Campbell LV: Obesity is an important determinant of baseline serum C-reactive protein concentration in monozygotic twins, independent of genetic influences. Circulation 2004, 109:3022-3028. 
15. Thorand B, Baumert J, Doring A, Herder C, Kolb H, Rathmann W, Giani G, Koenig W: Sex differences in the relation of body composition to markers of inflammation. Atherosclerosis 2006, 184:216-224.

16. Festa A, D'Agostino R, Williams K, Karter AJ, Mayer-Davis EJ, Tracy RP, Haffner SM: The relation of body fat mass and distribution to markers of chronic inflammation. International Journal of Obesity 2001, 25:1407-1415.

17. Deurenberg P, Van der Kooy K, Leenen R, Westrate JA, Seidell JC: Sex and age specific prediction formulas for estimating body composition from bioelectrical impedance: a cross validation study. Int J Obes 1991, 15:17-25.

18. Houtkooper BL, Lohman GT, Going BS, Howell HW: Why bioelectrical impedance analysis should be used for estimating adiposity. Am J Clin Nutr 1996, 64(suppl):436S-448S.

19. Roubenoff R: Applications of bioelectrical impedance analysis for body composition to epidemiologic studies. Am J Clin Nur 1996, 64(suppl):459S-462S

20. Chang PC, Li TC, Wu MT, Liu CS, Li Cl, Chen CC, Lin WY, Yang SY, Lin CC: Association between television viewing and the risk of metabolic syndrome in a community-based population. BMC Public Health 2008, 8:193.

21. Lin WY, Liu CS, Li TC, Lin T, Chen W, Chen CC, Li Cl, Lin CC: In addition to insulin resistance and obesity, hyperuricemia is strongly associated with metabolic syndrome using different definitions in Chinese populations: a population-based study (Taichung Community Health Study). Ann Rheum Dis 2008, 67(3):432-433.

22. Chen CC, Li TC, Chang PC, Liu CS, Lin WY, Wu MT, Li Cl, Lai MM, Li CC: Association among cigarette smoking, metabolic syndrome and its individual components: the metabolic syndrome study in Taiwan. Metabolism 2008, 57:544-548.

23. Chen CC, Li TC, Li Cl, Liu CS, Lin WY, Wu MT, Lai MM, Lin CC: The relationship between visfatin levels and anthropometric and metabolic parameters: association with cholesterol levels in women. Metabolism 2007, 56:1216-1220.

24. Lin CC, Liu CS, Li TC, Chen CC, Li Cl, Lin WY: Microalbuminuria and the metabolic syndrome and its components in the Chinese population. European Journal of Clinical Investigation 2007, 37(10):783-790.

25. Lin CC, Liu CS, Lai MM, Li Cl, Chen CC, Chang PC, Lin WY, Lee YD, Lin T, Li TC: Metabolic syndrome and its associated risk factors in a Taiwanese metropolitan adult population. BMC Public Health 2007, 7(1):739-743.

26. Bell NA, McClure PD, Hill RJ, Davies PSW: Assessment of foot-to-foot bioelectrical impedance analysis for the prediction of total body water. Eur J Clin Nutr 1998, 52:856-859.

27. Matthews DR, Hosker JP, Rudenski AS, Naylor BA, Treacher DF, Turner RC: Homeostasis model assessment: insulin resistance and beta-cell function from fasting plasma glucose and insulin concentrations in man. Diabetologia 1985, 28(7):412-419.

28. Grundy SM, Cleeman JI, Daniels SR, Donato KA, Eckel RH, Franklin BA, Gordon DJ, Krauss RM, Savage PJ, Smith SC Jr, Spertus JA, Costa F, American Heart Association; National Heart and Blood Institute: Diagnosis and management of the metabolic syndrome: an American heart Association/National Heart, Lung, and Blood Institute Scientific Statement. Circulation 2005, 112(17):2735-2752.

29. WHO: Definition, diagnosis and classification of diabetes mellitus and its complications: report of a WHO consultation. Geneva: World Health Organization 1999

30. Report of Department of Health: Department of Health definition and classification of obesity. Taiwan 2001.

31. DeLong ER, DeLong DM, Clarke-Pearson DL: Comparing the areas under two or more correlated receiver operating characteristic curves: a nonparametric approach. Biometrics 1988, 44:837-845.

32. Yudkin JS, Stehouwer CD, Emeis JJ, Coppack SW: C-reactive protein in healthy subjects: associations with obesity, insulin resistance, and endothelial dysfunction: a potential role for cytokines originating from adipose tissue? Arterioscler Thromb Vasc Biol 1999, 19:972-978.

33. Manns PJ, Williams DP, Snow CM, Wander RC: Physical activity, body fat, and serum C-reactive protein in postmenopausal women with and without hormone replacement. Am J Hum Biol 2003, 15:91-100.

34. Han TS, van Leer EM, Seidell JC, Lean MEJ: Waist circumference action levels in the identification of cardiovascular risk factors: prevalence study in a random sample. Br Med J 1995, 311:1401-1405.
35. Lean MEJ, Han TS, Morrison CE: Waist circumference as a measure for indicating need for weight management. Br Med J 1995, 311:158-161.

36. Lemieux S, Prud'homme D, Bouchard C, Tremblay A, Despres J-P: A single threshold value of waist girth identifies normal-weight and overweight subjects with excess visceral adipose tissue. Am J Clin Nutr 1996, 64:685-693.

37. Taylor RW, Keil D, Gold EJ, Williams SM, Goulding A: Body mass index, waist girth, and waist-to-hip ratio as indexes of total and regional adiposity in women: evaluation using receiver operating characteristic curves. Am J Clin Nutr 1998, 67(1):44-49.

38. Expert Panel on Detection and Treatment of High Blood Cholesterol in Adults: Executive summary of the third report of the National Cholesterol Education Program (NCEP) expert panel on detection, evaluation, and treatment of high blood cholesterol in adults (Adult Treatment Panel III). JAMA 2001, 285:2486-2497.

39. Alberti KG, Zimmet $P$, Shaw J: The metabolic syndrome-a new worldwide definition. Lancet 2005, 366(9491):1059-1062.

40. Chen J, Muntner P, Hamm LL, Jones DW, Batuman V, Fonseca V, Whelton PK, He J: The metabolic syndrome and chronic kidney disease in U.S. adults. Ann Intern Med 2004, 140:167-174.

41. Craig P, Colagiuri S, Hussain Z, Palu T: Identifying cut-points in anthropometric indexes for predicting previously undiagnosed diabetes and cardiovascular risk factors in the Tongan population. Obesity Research \&Clinical Practice 2007, 1:17-25.

\section{Pre-publication history}

The pre-publication history for this paper can be accessed here: http://www.biomedcentral.com/1471-2458/10/579/prepub

doi:10.1186/1471-2458-10-579

Cite this article as: Lin et al:: The relationship of high sensitivity Creactive protein to percent body fat mass, body mass index, waist-tohip ratio, and waist circumference in a Taiwanese population. BMC Public Health 2010 10:579.

\section{Submit your next manuscript to BioMed Central and take full advantage of:}

- Convenient online submission

- Thorough peer review

- No space constraints or color figure charges

- Immediate publication on acceptance

- Inclusion in PubMed, CAS, Scopus and Google Scholar

- Research which is freely available for redistribution

Submit your manuscript at www.biomedcentral.com/submit
C Biomed Central 